\title{
LOCAL NONDETERMINISM AND LOCAL TIMES OF GAUSSIAN PROCESSES ${ }^{1}$
}

\author{
BY SIMEON M. BERMAN
}

Communicated by H. Kesten, October 18, 1972

1. This work grew from a study of the conditions under which a Gaussian stochastic process has a "smooth" local time for almost all sample functions [1]-[4]. It is shown here that the main calculation in our previous work involves a property of Gaussian processes which is of independent interest-local nondeterminism. Let $X(t),-\infty<t<\infty$, be a Gaussian process with mean 0 , and $J$ an open interval on the $t$-axis. Suppose that

(1) $E[X(t)]^{2}>0$ and $E[X(t)-X(s)]^{2}>0, \quad$ for all $s$ and $t$ in $J$.

For arbitrary $t_{1}<\cdots<t_{m}$, where $t_{j} \in J$, form the ratio $V_{m}$ of the conditional to the unconditional variance:

$$
V_{m}=\frac{\operatorname{Var}\left[X\left(t_{m}\right)-X\left(t_{m-1}\right) \mid X\left(t_{1}\right), \ldots, X\left(t_{m-1}\right)\right]}{\operatorname{Var}\left[X\left(t_{m}\right)-X\left(t_{m-1}\right)\right]} .
$$

The numerator represents the error of prediction of $X\left(t_{m}\right)-X\left(t_{m-1}\right)$ based on $X\left(t_{1}\right), \ldots, X\left(t_{m-1}\right) . X$ is called locally nondeterministic on $J$ if

$$
\lim _{c \downarrow 0} \inf _{t_{m}-t_{1} \leqq c} V_{m}>0 \text {, for every } m \geqq 2 .
$$

This is a local version of the classical notion of nondeterminism: it signifies that an observation is "relatively unpredictable" on the basis of a finite set of observations from the immediate past.

We find conditions under which the members of certain classes of Gaussian processes are locally nondeterministic: for example, processes of multiplicity 1 , processes with stationary increments, and others.

2. Local nondeterminism means that there is an unremovable element of "noise" in the local evolution of the sample function. We expect such a function to be "locally irregular". And so it is: We show that local nondeterminism is one of the two main sufficient conditions in our result

AMS (MOS) subject classifications (1970). Primary 60G10, 60G15, 60G17; Secondary $60 \mathrm{G} 25$.

Key words and phrases. Local nondeterminism, local time, Gaussian process, multiplicity 1, stationary increments, sample function properties.

${ }_{1}^{1}$ This paper represents results obtained at the Courant Institute of Mathematical Sciences, New York University, under the sponsorship of the National Science Foundation Grant NSF-GP-28651. 
on the existence and smoothness of the local time of the sample functions of a general Gaussian process. This, in turn, implies the local irregularity of the sample functions themselves (nowhere differentiability, nowhere Hölder conditions of a given order, etc. [4]).

Let $X(t), 0 \leqq t \leqq T$, be a separable Gaussian process with Borel measurable sample functions. For every linear Borel set $A$, and $0 \leqq t \leqq T$, put

$$
v(A, t)=\text { Borel measure of } X^{-1}(A) \cap[0, t] .
$$

We say that the local time exists almost surely if, for almost every sample function, the measure $v(\cdot, t)$ is absolutely continuous with respect to Borel measure, for every $t$ in $[0, T]$. The local time is then defined to be the Radon-Nikodym derivative and is denoted $\phi(x, t)$.

THEOREM. Let $X(t)$ have mean 0 and satisfy the following conditions:

(I) $X(0)=0$, almost surely.

(II) $X$ is locally nondeterministic on $(0, T)$.

(III) There exist positive real numbers $\gamma$ and $\delta$ and a continuous even function $b(t)$ such that $b(0)=0, b(t)>0$ for $0<t \leqq T$, and

$$
\lim _{h \downarrow 0} h^{-\gamma} \int_{0}^{h}[b(t)]^{-1-2 \delta} d t=0,
$$

and

$$
E[X(t)-X(s)]^{2} \geqq b^{2}(t-s), \text { for all } s, t \text { in }[0, T] .
$$

Then there exists a version $\phi(x, t),-\infty<x<\infty, 0 \leqq t \leqq T$, of the local time which is jointly continuous in $(x, t)$ almost surely, and which satisfies a Hölder condition in $t$, uniformly in $x$, for every $\gamma^{\prime}<\gamma$ : There exist random variables $\eta$ and $\eta^{\prime}$ which are almost surely positive and finite such that

$$
\sup _{x}|\phi(x, t+h)-\phi(x, t)| \leqq \eta^{\prime}|h|^{\gamma^{\prime}}
$$

for all $s, t$ and $t+h$ in $[0, T]$, and all $|h|<\eta$.

This implies earlier results on Gaussian processes with stationary increments: Theorems $6.1,6.2$ and 6.3 of [3], and Theorem 4.1 of [4]. The stationary processes considered in [1] can be reduced to processes with stationary increments satisfying condition (I) above by considering the process $X(t)-X(0)$ in place of $X(t)$. Note that the only vestige of increment stationarity in the hypothesis of our theorem is the lower bound on the second moment in condition (III). Finally, we remark that condition (I) above is often no restriction on the process : If $X$ satisfies (II) and (III), then the process $X(t)-X(0)$ also satisfies (III), and will often satisfy (II). 
The proof of this theorem is a synthesis of earlier proofs of the same result for stationary processes and processes with stationary increments. Here we show that stationarity is not really important in the proof. The details of the proof, as well as other results on local nondeterminism, will be published elsewhere.

\section{REFERENCES}

1. S. M. Berman, Local times and sample function properties of stationary Gaussian processes, Trans. Amer. Math. Soc. 137 (1969), 277-299. MR 39 \# 1009.

2. - Harmonic analysis of local times and sample functions of Gaussian processes, Trans. Amer. Math. Soc. 143 (1969), 269-281. MR 40 \#2155.

3. - Gaussian processes with stationary increments: Local times and sample function properties, Ann. Math. Statist. 41 (1970), 1260-1272. MR 42 \#6916.

4. - Gaussian sample functions: uniform dimension and Hölder conditions nowhere, Nagoya Math. J. 46 (1972), 63-86.

Courant Institute of Mathematical Sciences, New York University, New York, NEW YORK 10012 